\title{
CLINICAL-EPIDEMIOLOGICAL PROFILE OF PATIENTS WITH TRIPLE-NEGATIVE BREAST CANCER AT INSTITUTO MÁRIO PENNA, BELO HORIZONTE, MINAS GERAIS
}

Ana Luiza de Freitas Magalhães Gomes'1, Marina Paixão de Madrid Whyte'1, Wagner Antonio Paz', Kerstin Kapp Rangel ${ }^{1}$, Paulo Guilherme de Oliveira Salles ${ }^{1}$

'Instituto Mário Penna - Belo Horizonte (MG), Brazil.

Introduction: Triple-negative breast cancer (TNBC) does not express estrogen and progesterone receptors, and does not overexpress the human epidermal growth factor 2. It represents $15 \%-20 \%$ of breast cancers and have worse prognosis, with scarce available therapies and overall survival (OS) of 18 months. For these particularities, research on TNBC is important for its greater understanding. Objectives: To describe the clinical-epidemiological profile of patients with TNBC at Instituto Mário Penna (IMP). To compare findings with data from the literature. Methods: Consultation of breast immunohistochemistry (IHC) performed at IMP between July/2012 and June/2017. TNBC were selected. Data were collected from patients in electronic medical records. Maximum follow-up until December/2018. Database and statistical analysis using the SPSS program. Bibliographic review used the key phrase: "triple-negative breast cancer". Results: 1,343 breast IHC performed at IMP in the studied period, 168 were TNBC (12.5\%). Mean age of 53.4 years. Mean follow-up of 41.7 months. Neoadjuvant chemotherapy (CT) performed in $46.4 \%$, with $12.8 \%$ of complete pathological response. Mean SG of 23.6 months, $20.2 \%$ progressed before the end of the treatment. Tumor mean size of $4.04 \mathrm{~cm}$. Mortality of $22 \%$, with $31.5 \%$ without information on death in the medical record, and about $17 \%$ on average with missing information. Table 1 shows the frequency distribution of the variables evaluated. Discussion: TNBC is a heterogeneous group of diseases, more commonly found in people aged under 40 years, of African descent, diagnosed at an advanced stage and with a high histological grade. Earlier metastasis, preferably visceral. More sensitive to CT, but with worse OS compared to other subtypes. Use of platinum, capecitabine and recent studies with immunotherapy are promising, in the search for better outcomes. Conclusion: The profile of patients with TNBC in IMP is compatible with that described in the literature. This study is a hypothesis generator and the basis for more complex research. High rates of missing information are a limiting factor. 